\title{
Reflets
}

Revue ontaroise d'intervention sociale et communautaire

\section{Rendez-vous « Santé globale »}

\section{Andrée Fauteux}

Volume 2, numéro 2, automne 1996

Vieillir à l'aube de l'an 2000

URI : https://id.erudit.org/iderudit/026133ar

DOI : https://doi.org/10.7202/026133ar

Aller au sommaire du numéro

Éditeur(s)

Reflets : Revue ontaroise d'intervention sociale et communautaire

ISSN

1203-4576 (imprimé)

1712-8498 (numérique)

Découvrir la revue

Citer cet article

Fauteux, A. (1996). Rendez-vous « Santé globale ». Reflets, 2(2), 109-117.

https://doi.org/10.7202/026133ar

Tous droits réservés (C) Reflets : Revue ontaroise d'intervention sociale et communautaire, 1996

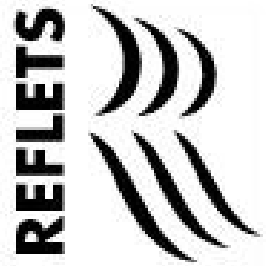

Ce document est protégé par la loi sur le droit d'auteur. L'utilisation des services d'Érudit (y compris la reproduction) est assujettie à sa politique d'utilisation que vous pouvez consulter en ligne.

https://apropos.erudit.org/fr/usagers/politique-dutilisation/ 


\title{
Rendez-vous «Santé globale»
}

\author{
Andrée Fauteux \\ Coordonnatrice, programme de maintien de l'autonomie, Centre de jour Guigues, \\ Ottawa
}

Ce court article traite d'un projet-pilote de prévention et de prise en charge par les aînées et les aînés avec objectif d'une plus grande autonomie.

De façon générale, avec l'âge, les problèmes de santé s'accroissent. Ils ont souvent pour effet de réduire l'autonomie, et par conséquent, la qualité de vie. Les personnes employées au Centre de jour Guigues sont régulièrement témoins de sérieux problèmes qui menacent l'autonomie des personnes âgées: surmédication, problèmes reliés à la tension artérielle, déficiences au plan alimentaire, maux de pied, manque d'activité physique, solitude ou signes de dépression, etc.

Depuis 1990, le Centre de jour Guigues se penche sur ces problèmes, en cherchant un moyen innovateur d'aiguiller les personnes âgées vers un programme de prévention et de promotion de la santé.

"Ce programme, basé sur la prise en charge de son bien-être par la personne âgée, adopte une approche holistique.»
Ce programme, basé sur la prise en charge de son bien-être par la personne âgée, adopte une approche holistique. Santé holistique, approche globale, autonomie, méthodes naturelles, médecine douce, etc. Que signifient ces nouveaux termes?

Ce sont des notions issues des mouvements sociaux et de santé des années 1960 et 1970, et il s'agit d'un regard renouvelé sur la santé afin de remplacer le point de vue dominant de la médecine scientifique moderne sur la maladie. La surmédication, l'usage excessif des tests, des interventions compliquées et coûteuses ont non seulement conduit à des dépenses extraordinaires pour la province, mais ont donné aux experts tous les 
pouvoirs. Ils ont aussi créé une dépendance excessive de la population par rapport au système de soins médicaux.

\section{But et objectifs du projet-pilote}

"Donner aux personnes âgées le pouvoir de décision sur leur vie, leur santé et leur bien-être, voilà le but recherché...»
Donner aux personnes âgées le pouvoir de décision sur leur vie, leur santé et leur bien-être, voilà le but recherché par le projetpilote «Rendez-vous Santé globale». Le projet vise à montrer que, grâce aux conseils, renseignements, services pratiques de professionnels en promotion du mieux-être et en prévention, les aînées et aînés peuvent adopter un mode de vie sain et équilibré. De plus, une approche globale des problèmes de santé peut réduire la fréquence des recours aux services médicaux traditionnels qui, comme nous l'avons déjà souligné, sont généralement coûteux.

Il s'agit, par le biais de ce projet, de permettre aux personnes âgées de rencontrer individuellement ou en groupe certains professionnels de la santé: massothérapeuthe, podologue, naturopathe, infirmière ou pharmacien, etc.

Les objectifs du projet pilote «Rendez-vous Santé globale» sont:

- promouvoir l'autonomie des ainées et aînés francophones d'Ottawa-Carleton;

- préserver leur santé;

- prolonger la durée de leur vie active;

- briser leur isolement et faciliter leur socialisation;

- assurer l'accessibilité du programme à des coûts abordables;

- créer un réseau de partenaires;

- promouvoir un climat de coopération au sein du personnel professionnel concerné par la santé et le mieux-être, et

- mettre au point des procédés permettant d'évaluer ce projet ou des projets analogues. 


\section{Étude des besoins}

En janvier 1995, le Centre de jour Guigues a reçu l'appui financier du ministère de la Santé du Canada, pour une durée de six mois, afin d'effectuer une étude des besoins. Cette étude, effectuée avec la méthode du sondage, a permis de recueillir les opinions et besoins de 239 francophones. Au total, $80 \%$ de femmes et $20 \%$ d'hommes ont été interrogés. Ils étaient âgés de 55 ans et plus et habitaient cinq municipalités de la région d'OttawaCarleton.

Ces personnes ont exprimé leurs préférences lorsqu'elles sont à la recherche d'aide au sujet de leur santé et de leur bien-être. Elles ont souligné l'importance de certains éléments, entre autres:

- la possibilité d'obtenir des services de santé en français;

- la présence en un même lieu, familier aux usagers, de représentants de diverses disciplines traditionnelles et non traditionnelles;

- l'importance de la prévention, de la prise en charge de son propre bien-être et de l'autodétermination, et,

- le besoin d'information sur la nature et les bienfaits des méthodes naturelles.

\section{Choix des aînées et aînés}

Dans l'étude, les personnes ont été interrogées sur les différents professionnels qu'elles aimeraient consulter. Le Tableau 1 illustre les professionnels choisis par les personnes âgées en vue d'obtenir des conseils ou des traitements de santé ${ }^{1}$. Dans ce tableau, il est intéressant de noter l'intérêt exprimé (les répondants pouvant choisir plus d'une réponse) pour rencontrer des professionnels traditionnels de la santé ainsi que des professionnels non traditionnels.

En ordre décroissant, les personnes âgées mentionnent les 
professionnels plus traditionnels suivants: podologue (43\%), nutritionniste (39\%),médecin (33\%), spécialiste en activité physique (26\%), pharmacien (17\%) et infirmière $14 \%{ }^{2}$.

Quant aux professionnels non traditionnels, les personnes âgées indiquent qu'elles aimeraient rencontrer les personnes suivantes: massothérapeute $(35 \%)$, naturopathe $(30 \%)$, chiropraticien (28\%), homéopathe (26\%), et réflexothérapeute (25\%).

Tableau 1 - Sondage Rendez-vous santé globale

Parmi les professionnels suivants, lesquels viendriez-vous rencontrer pour avoir des conseils et traitements pour votre santé?

(Les répondants pouvaient choisir plus d'une réponse.)
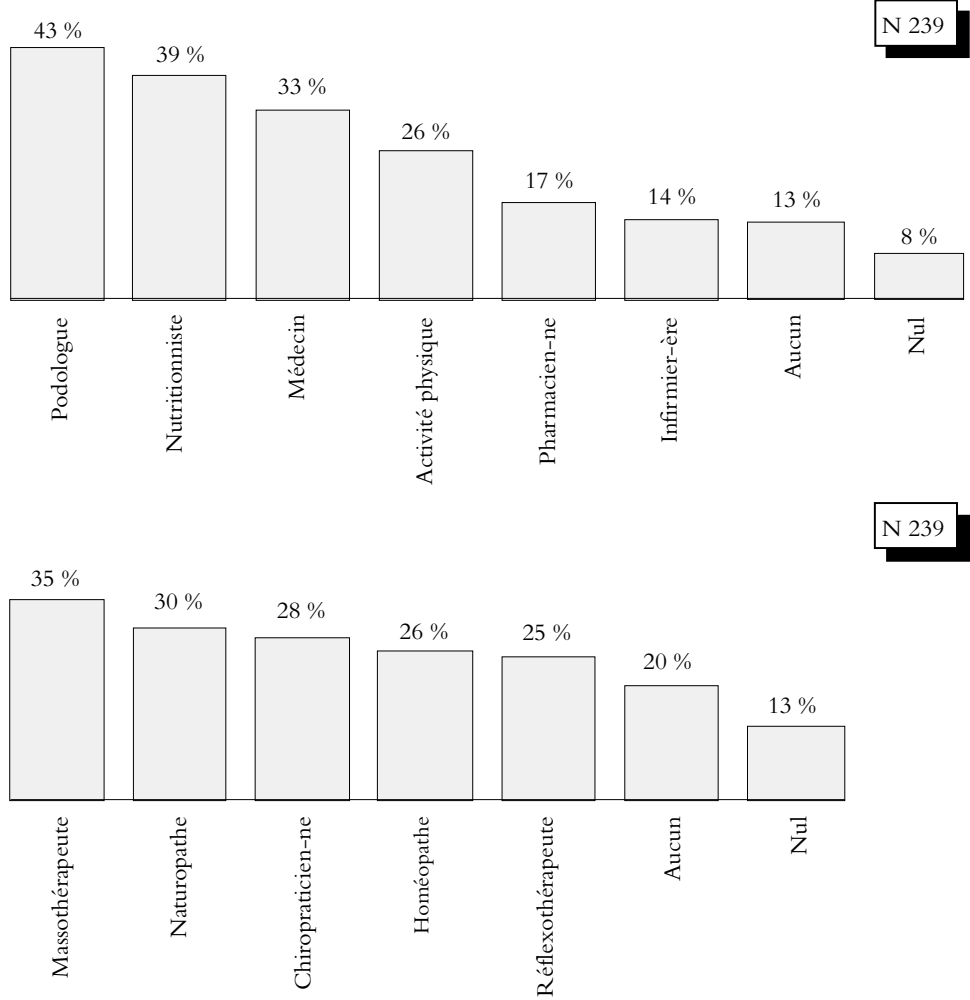
«Ces pourcentages... montrent bien l'intérêt des personnes âgées pour la médecine traditionnelle..."
Ces pourcentages, relativement similaires, montrent bien l'intérêt des personnes âgées pour la médecine traditionnelle comme pour la médecine non traditionnelle, et leur désir de rencontrer des professionnels dans l'une comme dans l'autre catégorie ${ }^{3}$.

Ce sont les aînées et aînés de 55 à 74 ans qui manifestent le plus d'intérêt pour les services complémentaires de professionnels de santé comme moyen de prévention et de promotion de la santé : massothérapeute, nutritionniste, podologue et naturopathe. Cette clientèle est aussi plus disposée à payer ce service et à se déplacer pour l'obtenir. Elle est également plus positive quant à l'impact du service sur la consommation des services traditionnels de santé.

Finalement, soulignons qu'en majorité les personnes âgées préferent le Centre de jour et le Centre de santé communautaire comme lieux de rencontre, et que $71 \%$ de l'échantillonnage s'intéressait à recevoir un plan personnalisé proposant des façons d'améliorer la qualité de vie.

\section{Partenariat}

La deuxième étape de l'étude consistait à consulter trente-et-une (31) agences de la région qui oeuvrent auprès des aînées et aînés francophones, afin d'obtenir leur avis sur l'utilité du projet et leur disponibilité pour établir, avec le Centre de jour Guigues, un partenariat qui assurerait le succès du projet-pilote.

Le Tableau 2 présente, par ordre d'importance, les professionnels et professionnelles de vingt-et-une (21) de ces agences qui pourraient donner des conseils et des soins aux aînés: conseillers en activité physique (100\%), médecin (100\%), pharmacien (100\%), nutritionniste $(95 \%)$, infirmière $(90 \%)$, podologue $(81 \%)$, chiropraticien $(81 \%)$, massothérapeute $(81 \%)$, naturopathe (81\%), réflexothérapeute (76\%), homéopathe (71\%). Dans la catégorie "autre», on mentionne les professionnels suivants: travailleur social, acupuncteur, physiothérapeute et ergothérapeute. 
Tableau 2 - Sondage Rendez-vous santé globale

\section{Parmi les professionnels suivants, lequel/lesquels, à votre avis, peut/peuvent donner aux aînés-ées des conseils/traitements utiles à leur santé?}

(Les répondants pouvaient choisir plus d'une réponse.)

\section{$\mathrm{N} 21$}

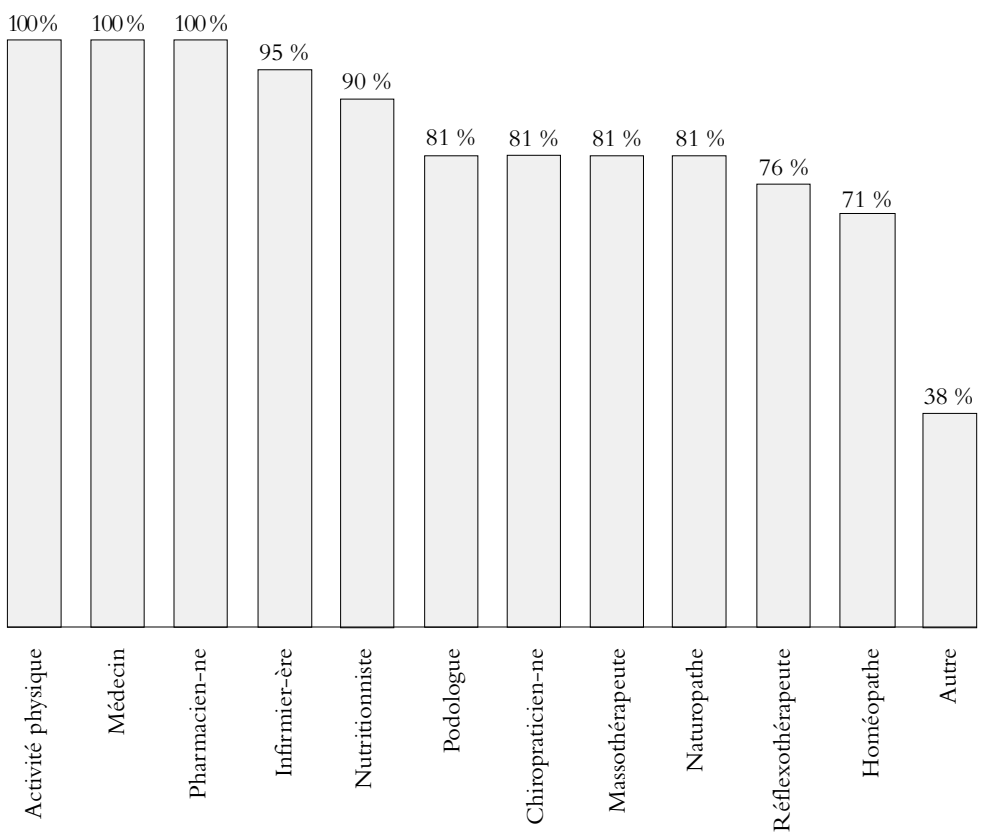

Dans l'ensemble de ces agences, $52 \%$ reconnaissent, en principe, le bien fondé du projet, pourvu que certaines conditions soient remplies :

- que le service soit décentralisé;

- que son développement se fasse en partenariat, et

- qu'il réponde aux besoins des personnes âgées.

Trente-trois pour cent (33\%) des agences offrent leur appui inconditionnel au projet. Les agences appuyant le projet offrent 
leur collaboration pour faire la promotion, prêter des locaux et du personnel de soutien.

\section{Innovations}

"...les aînées et aînés constitueront la majorité des membres du comité de direction qui prendra la relève...»

"...les professionnels de la santé sont de plus en plus conscients de l'importance d'une approche globale..."
Avec le projet-pilote, on tente certaines innovations. Ainsi, le Centre de jour Guigues est structuré de sorte que les usagers soient activement engagés dans la planification et la réalisation des projets. Ainsi, les aînées et aînés constitueront la majorité des membres du comité de direction qui prendra la relève du comité consultatif chargé de l'étude des besoins. Ces personnes seront impliquées dans le choix des professionnels de la santé, le système d'évaluation, la promotion du projet-pilote, le financement, etc.

Le projet-pilote "Rendez-vous Santé Globale» favorise et offre un modèle de coopération qui s'inscrit parfaitement dans la vision de partenariat communautaire entre usagers, agences et professionnels de la santé.

La responsable de l'étude de besoins a eu l'occasion de discuter du projet-pilote avec plus d'une cinquantaine de personnes pratiquant la médecine traditionnelle ou non traditionnelle, et avec des représentants d'agences qui offrent des services aux personnes âgées. L'intérêt et l'enthousiasme exprimés nous portent à croire que les professionnels de la santé sont de plus en plus conscients de l'importance d'une approche globale, de l'écoute et de la communication dans les rapports avec les usagers. Ils font ainsi écho aux principes directeurs du projet-pilote qui font appel au sens de la responsabilité de la personne envers sa santé, au respect de l'autodétermination de la personne, au développement de partenariats dans la communauté et à l'accessibilité des services des professionnels, à des prix abordables, en vue de la promotion du mieux-être et de la prévention. 


\section{Évaluation du projet-pilote}

Le comité de direction du projet-pilote s'assurera que les résultats du processus d'évaluation sont mis à la disposition des partenaires et de la communauté. Le caractère avant-gardiste de ce projet saura, du moins l'espérons-nous, susciter l'attention d'organisations francophones et anglophones qui offrent des programmes de prévention et de promotion de la santé.

\section{Mot de la fin}

Le projet a suscité de l'intérêt dans la communauté. Toutefois, il a également soulevé des questions concernant, entre autres, les points suivants :

- la duplication des services;

- une réponse aux besoins réels des aînées et aînés, et

- la complémentarité et la sécurité des services envisagés.

Il faut trouver réponse à ces questions. Par ailleurs, pour certains, l'approche semble viser davantage la dimension physique alors qu'il est fait mention de problèmes comme la dépression, la solitude, la toxicomanie, et les mauvais traitements qui menacent tout autant l'autonomie des aînées et aînés. Les services proposés ne toucheraient donc pas suffisamment la dimension de la santé mentale. De plus, l'environnement politique et économique actuel soulève l'interrogation sur l'opportunité de créer de nouveaux services. Ces points pertinents devront être pris en considération lors de l'implantation du service.

Le financement de ce projet n'est toujours pas assuré, mais le Centre de jour Guigues continue les démarches en ce sens, confiant que les bailleurs de fonds verront dans cette approche innovatrice un moyen supplémentaire de rationalisation des coûts des services de santé et de garantie du maintien de l'autonomie 
«...dans un tel projet repose une partie de la solution aux couts astronomiques de la santé.» chez les personnes âgées. Selon nous, dans un tel projet repose une partie de la solution aux coûts astronomiques de la santé. L'une des nombreuses causes est sans conteste le vieillissement de la population, mais il y a aussi la dépendance de la population envers l'État providence, habitude acquise au cours des trente dernières années. À l'aube de l'an 2000, où nous voulons compter sur des collectivités plus responsables et maintenir une bonne qualité de vie pour tous les groupes d'âge de la société, nous croyons, par ce projet, ouvrir la porte à de nouvelles façons de penser et de faire en matière de santé et de bien-être, afin de rendre les individus plus responsables d'eux-mêmes, de favoriser l'autonomie personnelle et la prise en charge de sa santé par la personne âgée.

\section{Notes}

1. Les répondantes et répondants pouvaient choisir plus d'une réponse.

2. Respectivement, $8 \%$ et $13 \%$ n'ont pas répondu à cette question.

3. Dans la catégorie «autre», les personnes âgées ont mentionné: acupuncteur, orthophoniste, psychologue, dermatologue, rhumatologue, ophtamologiste et physiothérapeute.

\section{Bibliographie}

CENTRE DE JOUR GUIGUES (1995). Rendez-vous Santé globale: rapport de l'étude de faisabilité, Ottawa, Centre jour Guigues. 